\title{
Amplified spontaneous emission-based technique for simultaneous measurement of temperature and strain by combining active fiber with fiber gratings
}

Cite as: Review of Scientific Instruments 73, 3369 (2002); https://doi.org/10.1063/1.1499758 Submitted: 11 December 2001 . Accepted: 06 June 2002 . Published Online: 22 August 2002

Y. C. Lai, G. F. Qiu, W. Zhang, L. Zhang, I. Bennion, and K. T. V. Grattan

\section{ARTICLES YOU MAY BE INTERESTED IN}

Fluorescence intensity ratio technique for optical fiber point temperature sensing Journal of Applied Physics 94, 4743 (2003); https://doi.org/10.1063/1.1606526

Remote thermometry with thermographic phosphors: Instrumentation and applications Review of Scientific Instruments 68, 2615 (1997); https://doi.org/10.1063/1.1148174

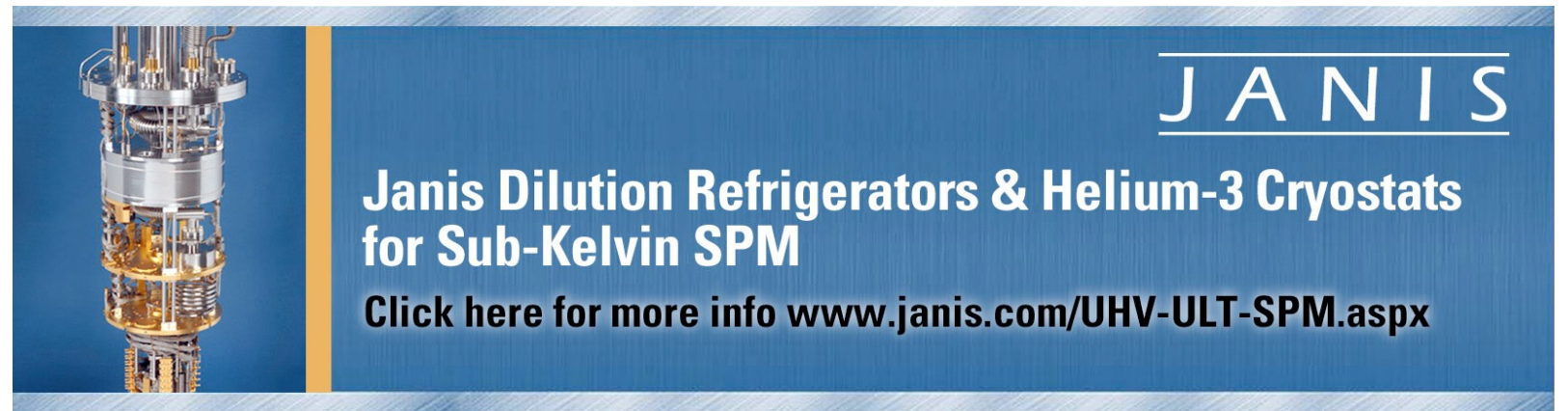




\title{
Amplified spontaneous emission-based technique for simultaneous measurement of temperature and strain by combining active fiber with fiber gratings
}

\author{
Y. C. Lai \\ Photonics Research Group, Aston University, Birmingham B4 7ET, United Kingdom \\ G. F. Qiu \\ School of Mechanical Engineering, Nanjing University of Science and Technology, Nanjing 210094, \\ People's Republic of China \\ W. Zhang, L. Zhang, and I. Bennion \\ Photonics Research Group, Aston University, Birmingham B4 7ET, United Kingdom \\ K. T. V. Grattan \\ Measurement and Instrumentation Centre, School of Engineering, City University, London EC1V OHB, \\ United Kingdom
}

(Received 11 December 2001; accepted for publication 6 June 2002)

This article reports on an optical fiber-based sensing system for carrying out the simultaneous measurement of temperature and strain. The sensor design is based on the combination of active doped optical fiber and fiber gratings. Erbium/ytterbium co-doped fiber is used to meet the requirements for both high temperature responsivity and small sensor size to address smart structure applications. The temperature dependence of the amplified spontaneous emission power under different pump wavelengths is used as the basis of the sensor and an optical reference to enhance the measurement resolution is discussed. The feasibility of this technique for simultaneous measurement of temperature and strain is demonstrated. (C) 2002 American Institute of Physics. [DOI: $10.1063 / 1.1499758]$

\section{INTRODUCTION}

"Smart structures" have seen a considerable level of interest in recent years, involving the use of advanced sensor technology and sophisticated signal processing. Optical fiber sensors have a role in the development and enhancement of this technology as compared to other, more conventional sensing approaches, and they show several important advantages in their operation. These include immunity to electromagnetic interference, high bandwidth, high sensitivity, and they have a high dynamic range and are lightweight. Additional advantages of this technology include their fatigue and corrosion resistance and the inherent strength of the glass fibers used. Further, the embedded optical fiber with a cladding diameter similar in value to that typical of modern communications fiber does not appear to degrade the static mechanical properties of the host. The optical fiber itself is widely known for its use as a communications medium and thus the combination of optical fiber technology and smart structures enables the realization of multiplexed arrays of the fiber sensors, interconnected by other fibers to communicate the signals, and with that achieve the implementation of quasidistributed sensors. A fiber Bragg grating (FBG) sensor system is an excellent example of this, however, the intrinsic response of any individual FBG to either strain or temperature is similar, in each case, to producing a wavelength shift of the grating. This is significant in quasistatic strain sensing as the response to any temperature variation along the fiber is indistinguishable from the effect of the strain (indeed the fiber is more sensitive to temperature than strain), which complicates its application in sensor systems.

It is important that methods are developed to distinguish these effects in the signal received from the grating. Different techniques have been proposed to discriminate the above parameters including using a reference fiber grating, ${ }^{1}$ a fiber grating pair with different sensitivities to temperature and strain, ${ }^{2-4}$ and the combination of a fiber grating with other fiber devices. ${ }^{5,6}$ Erbium-doped fiber itself has been reported as an intrinsic temperature sensor. ${ }^{7}$ Recently, the technique of combining an active fiber with a fiber grating to achieve simultaneous temperature and strain measurement has been considered. ${ }^{8}$ This method offers a larger temperature measurement range than other techniques, due to the intrinsic temperature-dependent power characteristics of the amplified spontaneous emission (ASE) in the erbium-doped or erbium/ ytterbium co-doped fiber used. In the cases reported, however, a long length of active fiber has to be used to enhance the responsivity, which is not a practical solution for quasidistributed measurement in smart structures, and the resolution achieved for the temperature measurement was poor.

In this article an investigation of a scheme to measure the strain and temperature simultaneously using an ASEbased approach is reported. The key advantage over the approaches previously discussed lies in the amplification offered by the system, thus enhancing the measuranddependent signal and taking greater advantage of the nature 


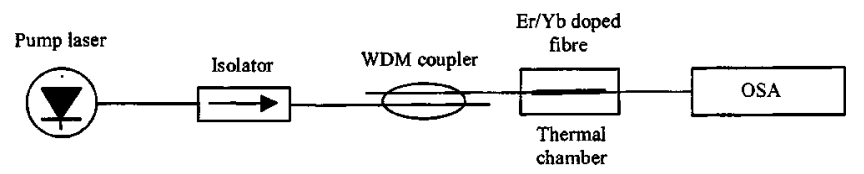

FIG. 1. Schematic of the experimental arrangement for investigating the ASE power. WDM coupler: wavelength division multiplexing coupler; OSA: optical spectrum analyzer.

of the doped-fiber optical system. The use of optical signal amplification is designed to ensure a better signal-to-noise ratio in the sensor system.

\section{PRINCIPLE OF OPERATION}

To recover both temperature and strain without ambiguity, in most work a two-grating sensing system is used to describe the relationship between the wavelength shifts and these measurands. These two gratings should have different sensitivities to either of the measurands to make the determinant of the response matrix be nonzero. Often, one grating is strain-isolated or stabilized at a fixed temperature, in both cases requiring a complex arrangement. The strength of the approach in the work herein is that instead of a two-grating system, a single grating can be used to encode the strain and temperature if another parameter, in addition to the Bragg wavelength, is available. Using an active fiber can provide information on the optical power at the Bragg wavelength and in the work the ASE power of the erbium or erbium/ ytterbium doped fiber used is monitored and is temperature dependent. The power variation of the ASE $\Delta P$ and the Bragg wavelength shift $\Delta \lambda_{B}$ can be expressed by

$$
\left(\begin{array}{c}
\Delta P \\
\Delta \lambda_{B}
\end{array}\right)=\left[\begin{array}{ll}
A & B \\
C & D
\end{array}\right]\left(\begin{array}{c}
\epsilon \\
\Delta T
\end{array}\right),
$$

where $\epsilon$ is the applied strain and $\Delta T$ is the temperature change. The two measurands can be determined simultaneously by measuring the power change and the Bragg wavelength shift, and this is the approach used in this article. This approach complements that described by some of the authors, where the spontaneous emission alone from the fiber is used, and either the fluorescent lifetime or the ratio of the emission from two coupled energy levels is determined, and monitored as the temperature-dependent parameter. The key advantage of the system design is the use of the optical amplification of the signal received.

\section{EXPERIMENT AND RESULTS}

\section{A. ASE power}

The temperature dependence of the ASE power of the active fiber was first investigated. A schematic diagram of the experimental arrangement is shown in Fig. 1. The system comprises a pump diode laser, the output of which is coupled through an optical isolator to a wavelength division multiplexing (WDM) coupler. The light then enters the $\mathrm{Er} / \mathrm{Yb}$ doped fiber under test, which is mounted in a stable oven for temperature tests to be carried out. For smart structure applications, a short length of sensing device is highly desirable for distributed or quasidistributed measurement to enable a
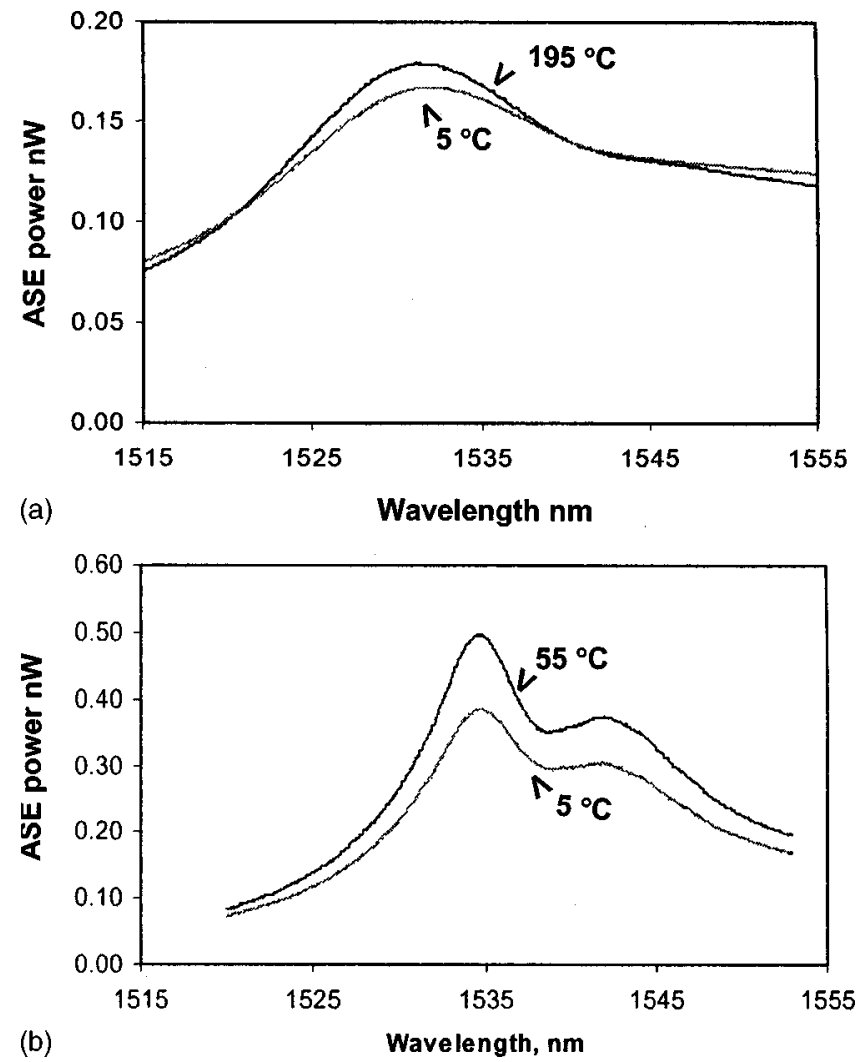

FIG. 2. ASE power spectra of $6.5 \mathrm{~cm} \mathrm{Er} / \mathrm{Yb}$ codoped fiber pumped by (a) $980 \mathrm{~nm}$ laser diode (temperature range 5 to $195^{\circ} \mathrm{C}$ ); (b) $1480 \mathrm{~nm}$ laser diode (temperature range 5 to $55^{\circ} \mathrm{C}$ ).

closer location of the strain region under study. In the experiment carried out, a piece of $\mathrm{Er} / \mathrm{Yb}$ co-doped fiber with a length of $6.5 \mathrm{~cm}$ was used and the thermal chamber was designed and used to apply the desired temperature to the active fiber, with a temperature stability of $0.1^{\circ} \mathrm{C}$. The ASE power from the system was captured by an optical spectrum analyzer (OSA) and the temperature dependence of the measured ASE power values, when pumped at wavelengths of both 980 and $1480 \mathrm{~nm}$, were determined. This enabled any effects of pump wavelength to be evaluated.

Several ASE power spectra thus obtained are shown in Fig. 2. Figure 2(a) represents the ASE power spectra obtained when the system was pumped by a $980 \mathrm{~nm}$ laser diode while the temperature was varied from 5 (lower curve) to $195^{\circ} \mathrm{C}$ (upper curve), and Fig. 2(b) shows results from a fiber pumped by a $1480 \mathrm{~nm}$ laser diode while the temperature was varied from 5 (lower curve) to $55^{\circ} \mathrm{C}$ (upper curve). Clearly the latter gives a much higher temperature responsivity showing a greater change over a smaller temperature region of study. In both cases, the output peaks at a wavelength of $\sim 1535 \mathrm{~nm}$, as would be expected from the presence of the $\mathrm{Er}^{3+}$ ion.

The ASE power shows a different spectral profile and temperature dependence when pumped at different wavelengths. When excited by the $1480 \mathrm{~nm}$ laser diode used, the ASE power observed over all the wavelengths increases, as the applied temperature increases. The maximum change appears at $\sim 1535 \mathrm{~nm}$. When pumped by the $980 \mathrm{~nm}$ laser diode, the overall ASE power seen for this pumping source 


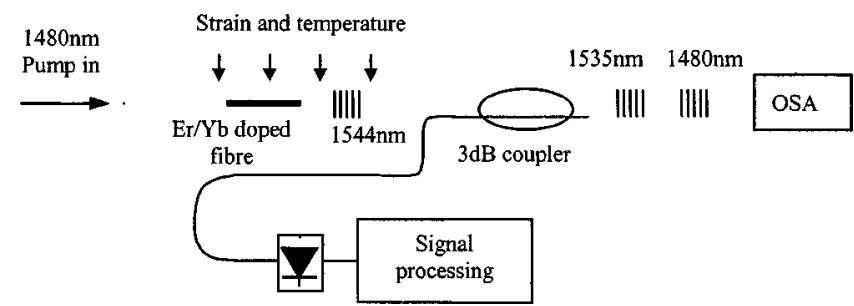

FIG. 3. Experimental setup for simultaneous measurement of temperature and strain. OSA: optical spectrum analyzer.

shows almost no change over a temperature change of $180^{\circ} \mathrm{C}$. However, the ASE power spectrum shows some interesting features. Around $1530 \mathrm{~nm}$, the power decreases with the temperature where it shows its maximum temperature responsivity. Beyond that, the temperature responsivity reduces as the wavelength deviates from $1530 \mathrm{~nm}$, and changes its sign at two specific wavelengths, as can be seen from the spectrum illustrated. It may be observed from Fig. 2(a) that at two specific wavelengths $(\sim 1520$ and $1540 \mathrm{~nm})$, the ASE power does not vary with the temperature. Therefore, the power at these two wavelengths may be used here to create a reference wavelength as a result, to remove any uncertainty caused by the ASE power fluctuation and the optical transfer function variation.

The temperature responsivities near the peak outputs, at $1530 \mathrm{~nm}$ for $980 \mathrm{~nm}$ pumping and at $1535 \mathrm{~nm}$ for $1480 \mathrm{~nm}$ pumping were measured to be 0.0018 and $0.0218 \mathrm{dBm} /{ }^{\circ} \mathrm{C}$, respectively. Therefore, the longer wavelength $1480 \mathrm{~nm}$ pumping is preferable for practical systems, to obtain a larger responsivity and a higher resolution, and thus was used in this work.

\section{B. Temperature and strain measurement}

The arrangement used in this work for a temperature and strain measurement system is shown schematically in Fig. 3. The temperature measurement was implemented by measuring the ASE power change around $1535 \mathrm{~nm}$ where the maximum temperature responsivity is seen. A fiber grating with a central wavelength of $1535 \mathrm{~nm}$, and a bandwidth of $\sim 1 \mathrm{~nm}$ was placed behind a $3 \mathrm{~dB}$ coupler so that the reflection of the grating represents the ASE power level. Under these wavelength and bandwidth conditions, the temperature responsivity is $0.0217 \mathrm{dBm} /{ }^{\circ} \mathrm{C}$, essentially the same as the maximum responsivity measured previously.

The ASE power level reflected by the fiber grating was determined as a function of the temperature of the oven used to simulate its performance over a short temperature range. The result of this study is depicted in Fig. 4. From this, it can be seen that there is a close correlation and an effective linear relationship between the ASE power level obtained and the temperature variation. This is a useful performance feature for sensor applications, and makes the implementation of a correction for temperature compensation of the strain measurement relatively easy.

The resolution of the temperature measurement is limited by the ASE power fluctuation and the varying optical transfer function. Since the active fiber operates in small sig-

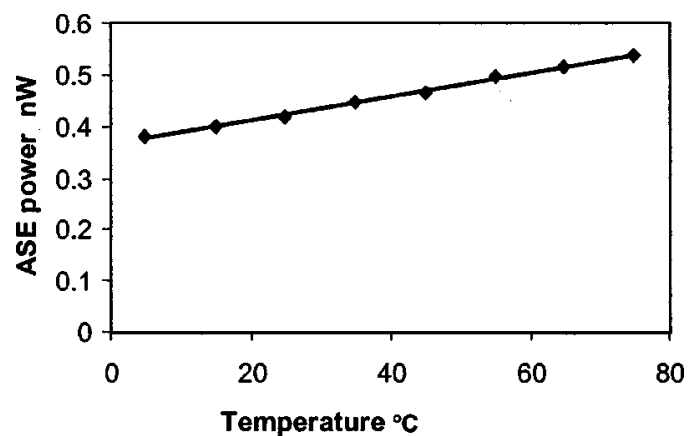

FIG. 4. Performance of the ASE-based temperature sensor system, showing reflection at $1535 \mathrm{~nm}$ vs temperature.

nal gain mode, there still remains some pump power unabsorbed, as is shown in Fig. 5 where the power is plotted as a function of wavelength. It was noticed that this residual power level at $1480 \mathrm{~nm}$ does not vary with temperature. As a consequence, this feature may also be used as a reference mechanism to normalize the temperature-dependent ASE power. In the experiment carried out, a fiber grating with its wavelength centered at $1480 \mathrm{~nm}$ was used to detect the residual pump power. The optical signals reflected from the two gratings thus incorporated in the system were detected separately, and the signals processed. The resolution for the temperature measurement thus obtained was $0.3^{\circ} \mathrm{C}$, a satisfactory result for many practical sensor applications.

\section{Bragg grating and doped fiber combination}

The use of the ASE technique with a FBG was simulated in the following test. A fiber Bragg grating with a central wavelength of $1544 \mathrm{~nm}$ and a length of $1 \mathrm{~cm}$ was spliced close to the $\mathrm{Er} / \mathrm{Yb}$ doped fiber, with the Bragg wavelength being affected by both strain and temperature. The associated Bragg wavelength shift was monitored by the use of an OSA although in a practical smart structure, one of a number of dedicated monitoring schemes discussed in the literature could be used. ${ }^{9}$ These are not discussed further herein. Since the temperature variation can be determined by measuring the ASE power level, it has been shown that the simultaneous measurement of temperature and strain can be readily achieved. The change in the center wavelength of the FBG is observed and the temperature determined from the calibrated

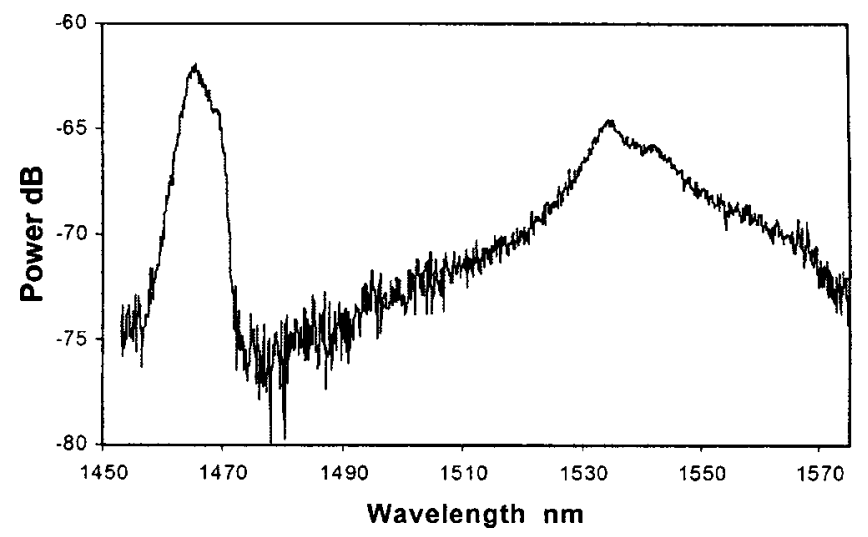

FIG. 5. ASE power and residual pump power. 
ASE power signal. The temperature correction is applied to the FBG wavelength shift and thus the magnitude of the strain effect can be deduced from the calibration of strain versus wavelength shift alone. The performance compares well to that of other systems using spontaneous emission, in an unamplified form. ${ }^{10}$ It provides an alternative, especially in situations where the unamplified spontaneous signal is weak, and therefore, the resulting measurement would be subject to noise, increasing the uncertainty of the temperature measurement. This approach may be particularly valuable in applications where the monitoring system needs to be remote, e.g., in an oil well or in a furnace lining where, for safety and ease of use, the control electronics and data processing system may be some distance, even kilometers away.

\section{DISCUSSION}

A technique has been demonstrated which is capable of the simultaneous measurement of temperature and strain in which $\mathrm{Er} / \mathrm{Yb}$-doped fiber has been used to provide a high temperature responsivity. The size of the sensor thus obtained is significantly reduced over alternatives, enabling it to be a candidate for effective quasidistributed sensing in smart structure applications. The temperature range over which it has been demonstrated in this work has been comparatively modest, but representative for major applications. However, previous work by some of the authors ${ }^{11}$ has shown the potential for the use of doped fiber-based systems and the measurement of the subsequent fluorescence emission for temperature monitoring up to $\sim 1000{ }^{\circ} \mathrm{C}$. Work is continuing to determine the effective limits, in temperature terms, of this approach. In addition to the measurement of temperature and strain, the fiber grating has also been used to overcome any ASE power fluctuations and optical transfer function variations. A higher resolution and measurement range for the temperature determination can be expected. Further results will be reported from continuing work.

\section{ACKNOWLEDGMENT}

The authors at City University are pleased to acknowledge the support of EPSRC via the INTErSECT Faraday Partnership and other initiatives.

${ }^{1}$ A. D. Kersey, T. A. Berkoff, and W. W. Morey, Opt. Lett. 18, 72 (1993).

${ }^{2}$ M. G. Xu, J. L. Archambault, L. Reekie, and J. P. Dakin, Electron. Lett. 30, 1085 (1994)

${ }^{3}$ S. E. Kanellopoulos, V. A. Handerek, and A. J. Rogers, Opt. Lett. 20, 333 (1995).

${ }^{4}$ H. J. Patrick, G. M. Williams, A. D. Kersey, J. R. Pedrazzani, and A. M. Vengsarkar, IEEE Photonics Technol. Lett. 8, 1223 (1996).

${ }^{5} \mathrm{H}$. Singh and J. Sirkis, Conference Proceedings OFS-11, Japan, May, 1996, pp. 108-111.

${ }^{6}$ X. D. Jin and J. Sirkis, Proc. SPIE 3042, 120 (1997).

${ }^{7}$ E. Maurice, G. Monnom, B. Dussardier, A. Saissy, D. B. Ostrowsky, and G. W. Baxter, Appl. Opt. 34, 8019 (1995).

${ }^{8}$ J. Jung, N. Park, and B. Lee, Appl. Opt. 39, 1118 (2000).

${ }^{9}$ A. Orthonos, in Optical Fiber Sensor Technology, edited by K. T. V. Grattan and B. T. Meggitt (Kluwer Academic, Dordrecht, 2000), pp. 79187.

${ }^{10}$ K. T. V. Grattan, Z. Y. Zhang, T. Sun, Y. Shen, L. Tong, and Z. Ding, Meas. Sci. Technol. 12, 981 (2001)

${ }^{11}$ S. A. Wade, D. I. Forsyth, K. T. V. Grattan, and Q. Guofu, Rev. Sci. Instrum. 72, 3186 (2001). 